\title{
BROWN SUGAR. Tendenzen IM neueren Rhythm'n'Blues
}

\author{
Martin Pfleiderer
}

1998 veröffentlichte Lauryn Hill ihre Debüt-CD The Miseducation Of Lauryn Hill. Hill war bereits durch kleinere Filmrollen und als Mitglied des RapTrios The Fugees bekannt geworden. In The Miseducation Of Lauryn Hill sind jedoch nur wenige Raps zu hören, stattdessen zahlreiche Gesangsnummern, die in der Tradition des Soul und Rhythm'n'Blues stehen. Umso mehr überrascht, dass das Album sowohl ein großer kommerzieller Erfolg wurde als auch ein breites Medienecho auslöste. ${ }^{1}$ Im Februar 1999 wurden der damals 23-jährigen Hill für das Album fünf Grammys in den Kategorien Album of the Year, Best New Artist, Best Female R\&B Vocal Performance, Best R\&B Song und Best R\&B Album verliehen - ein Erfolg, den vor ihr keine andere Künstlerin erzielt hatte. Offensichtlich lebte in den späten 1990er Jahren parallel zum Höhenflug des HipHop und zum Erfolg von afroamerikanischen Pop-Stars die Tradition der Soul-Musik wieder auf - zeitweise wurde gar von einem »Neo-Soul«-Stil gesprochen:

»In the 90s and early 2000s, the term neo-soul has been used to describe urban contemporary artists who have one foot in the past and one foot in the present - the past being the classic soul of the 60s and 70s, the present being urban and hip-hop« (Henderson 2003: 855).

Vergleicht man den R\&B der vergangenen zehn Jahre mit der afroamerikanischen Musik der 1960er Jahre oder mit den Entwicklungen der Rap Music, so sind die musikalischen Veränderungen wenig spektakulär. Allerdings lassen sich gegenüber der afroamerikanischen Musik der vorangegangenen Dekaden durchaus einige Neuerungen feststellen. Zum einen vollzieht sich eine Annäherung an musikalische Gestaltungsmittel und Produktionsweisen der Rap Music, die durch zahlreiche gemeinsame Produktionen von Künstlern und

1 Das Album führte 1998 die Jahresbestenliste zahlreicher Musikkritiker an und Hill wurden mehrere Cover-Stories in US-amerikanischen Zeitschriften wie Time, Esquire u.a. gewidmet. 
Produzenten aus den Bereichen R\&B und Rap Music unterstützt wird. Zum anderen beziehen sich R\&B-Sängerinnen und -Sänger in ausgeprägter Weise auf die Tradition der populären afroamerikanischen Musik. Sie knüpfen vor allem an den Soul der 1960er und 1970er Jahre an und beziehen sich in ihrem Gesangsstil darüber hinaus auf die Gesangstradition der afroamerikanischen Gospel Music. Angesichts der Vielzahl und Vielfalt der R\&B-Produktionen und -Künstler der letzten zehn Jahre ist es mir nicht möglich, ein umfassendes Bild des zeitgenössischen R\&B zu zeichnen. ${ }^{2}$ Vielmehr möchte ich mich auf die Darstellung von drei Tendenzen beschränken: auf die angedeutete Wechselbeziehung zwischen R\&B und Rap Music, sodann am Beispiel Lauryn Hills auf den melismatischen Gesangsstil der R\&B-Vokalisten und schließlich anhand eines Cover-Textes des Neo-Soul-Stars D'Angelo (Michael Eugene Archer) auf die Art und Weise, wie sich die jungen, zumeist in den 1970er Jahren geborenen afroamerikanischen Künstler innerhalb der heutigen afroamerikanischen »Post-Soul«-Kultur (George 1992, Neal 2002) positionieren.

Zuvor erscheint mir jedoch eine Erläuterung des Ausdrucks R\&B bzw. »Rhythm'n'Blues« notwendig. Seitdem er im Jahre 1949 von der USamerikanischen Musikindustrie als Bezeichnung für Musikrichtungen, die sich an das Marktsegment der afroamerikanischen Musikhörer richten, lanciert wurde und dabei das nach dem zweiten Weltkrieg als anachronistisch empfundene Label »Race Music« ablöste, fungiert »Rhythm'n'Blues« als eine relativ offene Sammelbezeichnung für unterschiedliche Stilausprägungen populärer afroamerikanischer Musik. Bereits in den 1950er Jahren war das Ziel vieler R\&B-Produktionen nicht nur der Erfolg bei der schwarzen, sondern ebenso bei der weißen Käuferschaft des Mainstream-Pop-Marktes. Nachdem der Crossover in den Pop-Markt zunächst durch Cover-Versionen weißer Künstler und sodann durch einzelne afroamerikanische Rock'n'RollStars (Fats Domino, Chuck Berry, Little Richard) erreicht wurde, war in den 1960er Jahren die afroamerikanische Plattenfirma Motown Records mit Gruppen wie den Supremes oder den Four Tops im Pop-Markt weißer Jugendlicher erfolgreich (vgl. Brackett 1994). Die Grenzen zwischen afroamerikanischem R\&B und Pop sind seither fließend. Viele afroamerikanische Künstler streben den Erfolg in beiden Marktsegmenten an und die großen Plattenfirmen haben spätestens seit der Studie zur Marktentwicklung populärer Musik, die Anfang der 1970er von der Harvard University Business School unter dem Titel A Study of Soul Music Environment im Auftrag von Columbia Records erstellt wurde (vgl. George 1988: 135-138; Bynoe 2002:

2 Einen weitgehend journalistisch gehaltenen Überblick über die Vielfalt des R\&B geben die Soul- und R\&B-Lexika von Bogdanov et al. (2003) und Larkin (1998). 
223-225), das Erfolgspotential afroamerikanischer Musik für den weißen Pop-Markt erkannt.

Im Zuge der allgemeinen Politisierung durch die afroamerikanische Bürgerrechtsbewegung in den 1960er Jahren, die auch von vielen Musikern unterstützt wurde (vgl. Ward 1998), erfolgte die Umbenennung der »R\&B«Charts in Billboard, dem Branchenorgan der US-amerikanischen Tonträgerindustrie, in »Soul« im Jahre 1969 und schließlich 1982 in »Black Music«. Die Bezeichnung »Black Music «, die sich auf das »black consciousness«, das neue Selbstbewusstsein der Schwarzen in den USA bezieht, wurde allerdings von den Plattenhändlern als zu stark ethnisch eingegrenzt und somit als verkaufshemmend empfunden, so dass seit 1990 wieder auf die neutrale und unpolitische Bezeichnung »Rhythm'n'Blues« zurückgegriffen wird (vgl. George 1992: 1); innerhalb der Plattenindustrie sind jedoch auch andere Kategorien verbreitet wie z.B. »urban« oder »urban contemporary«. Inzwischen werden in Billboard »R\&B« und »HipHop« zu einer gemeinsamen Charts-Kategorie zusammengefasst. Allerdings lassen sich die beiden Genres eindeutig voneinander unterscheiden, da entweder Gesang oder aber Sprechgesang (Rap) im Zentrum steht. Im Folgenden verstehe ich unter »Rhythm'n'Blues « die Musik von afroamerikanischen Sängerinnen und Sängern, die zumindest in den USA vorwiegend für einen afroamerikanischen Markt produziert wird.

Die 1990er Jahre waren eine goldene Zeit der Rap Music. HipHop-Bands wie Public Enemy, NWA und DeLa Soul sorgten bereits vor 1990 für Aufsehen; es folgten Rap-Stars wie Snoop Doggy Dogg, Tupac Shakur und Notorious B.I.G., durch deren Erfolg Rap Music allmählich zu einem MainstreamMusikgenre avancierte. Die Hochphase des HipHop hängt eng mit Veränderungen in der musikalischen Gestaltung zusammen, die wiederum mit neuen Produktionstechniken in Verbindung stehen. Joseph Schloss beschreibt in seiner Studie Making Beats. The Art of Sample-Based Hip-Hop (2004), wie Ende der 1980er Jahre der Einsatz von preiswerteren Sampling-Geräten zu einer neuen Produktionsweise und einem neuen Produzententyp innerhalb der Rap Music geführt hat. Nicht mehr die DJs, sondern Produzenten und Produzenten-Teams wie Bomb Squad (Hank Shocklee, Keith Shocklee, Eric Sadler und Chuck D./Carlton Ridenhour) bei Public Enemy, Dr. Dre bei NWA oder Prince Paul bei DeLa Soul zeichneten nun für das Klanggeschehen verantwortlich, vor allem für die so genannten Beats, also das rhythmische Gerüst der Stücke, über die dann die MCs rappten. Anders als beim DJ-ing mit zwei Plattenspielern können mit Samplern und Software-Sequenzern zahlreiche Klangbausteine aus mehreren unterschiedlichen Quellen zu einem Beat zusammengesetzt werden (vgl. Schloss 2004: 105ff.). Dadurch werden 
die Beats abwechslungsreicher und die Klangtexturen z.B. bei Public Enemy dichter als in der Rap Music aus der Zeit vor dem Sampling, als neben DJ-ing Rhythmusmaschinen und Synthesizer die Klangproduktion dominierten.

Die neuen HipHop-Produzenten sind in der Regel selbst Musiker oder Rapper. Und viele von ihnen produzieren Beats sowohl für Rap-Aufnahmen als auch für R\&B-Songs, so dass sich im Laufe der 1990er Jahre die Klanggestalten der beiden Genres zum Teil einander angenähert haben. Dies lässt sich am Beispiel einiger Produzenten festmachen, so bereits an Andre Harrell und Teddy Riley. Harrell, der in den 1980er Jahren als Dr. Jekyll des Rap-Duos Dr. Jekyll und Mr. Hyde bekannt geworden war, begann parallel zu seiner künstlerischen Karriere 1983 bei dem führenden HipHop-Label Def Jam als stellvertretender Leiter der A\&R-Abteilung zu arbeiten, bevor er 1986 Uptown Entertainment gründete. ${ }^{3}$ Gemeinsam mit dem Sänger, Songwriter und Produzenten Teddy Riley popularisierte Harrell Ende der 1980er den »New Jack Swing «, eine Verbindung von synthetischen Funk-Beats, wie sie vor der Einführung der Sampling-Technik im HipHop üblich waren, und R\&B-Gesang - etwa mit Rileys eigener Band Guy (»Guy«, 1988), mit Keith Sweat (»I Want Her«, 1987) oder mit Bobby Brown (»Don't Be Cruel«, 1988). Sean Combs, der nach seinem Betriebswirtschafts-Studium an der HowardUniversity in Washington ab 1989 bei Harrells Uptown Entertainment als A\&R-Mananger arbeitete, führte diese Idee weiter und produzierte mit der »Queen of HipHop Soul« Mary J. Blige (»What's The 411«, 1992, und »My Life«, 1994) eine erfolgreiche Verbindung von aktuellen HipHop-Beats und Soul-Gesang. Sean Combs, der später unter den Künstlernamen Puff Daddy und P-Diddy auch selbst als Rapper aktiv wurde, gründete 1995 die Firma Bad Boy Entertainment, bei der er sowohl Rap- (Heavy D., Notorious B.I.G.) als auch R\&B-Künstler produzierte.

Im Laufe der 1990er Jahren verschwammen die Grenzen zwischen Rap Music und R\&B zunehmend. Einerseits zeigen viele Rap-Stücke starke Einflüsse des R\&B, sowohl durch den R\&B-Gesang des Refrains als auch durch Beats, deren Samples R\&B-Aufnahmen entnommen wurden. Andererseits greifen R\&B-Künstler in ihren Stücken auch auf typische, mit der SamplingTechnik produzierte HipHop-Beats und auf Rap-Einlagen zurück. Das »produced by « wird in beiden Genres zum zentralen Markenzeichen. So nennt beispielsweise Mary J. Blige auf einem Aufkleber auf der CD-Hülle von »No More Drama (2001) zahlreiche Produzenten, die Stücke zum Album beigesteuert haben, u.a. Dr. Dre, die Neptunes, Kenny Flav und Missy Elliott. Missy Elliott und Timbaland wiederum protegieren mit Tweet (Charlene

31995 ging Harrell zu Motown Records und verkaufte seinen Anteil an Uptown Entertainment für 40 Millionen US-Dollars (vgl. Bynoe 2002: 228f.). 
Keys) eine junge R\&B-Sängerin, deren Songs sie die charakteristischen, von Timbaland produzierten Beats unterlegen (»Southern Hummingbird«, 2002). Aktuelles Beispiel für einen Produzenten und Rapper ist Kanye West, der sowohl die Musik des Rap-Künstlers Common als auch die Debüt-CD des jungen R\&B-Sängers John Legend produziert.

Ebenfalls im Zusammenhang mit den neuen Produktionsweisen mit Samplern und Software-Sequenzern stehen die zahlreichen Eigenproduktionen im R\&B-Bereich seit den späten 1990er Jahren insbesondere von Künstlern, die dem Neo-Soul zugerechnet werden. Sowohl das erfolgreiche Album von Lauryn Hill als auch die zweite CD von D'Angelo (Voodoo, 2000) wurden von den Künstlern selbst produziert. Die neue Produktionstechnologie macht Eigenproduktionen nicht mehr nur für einen finanzstarken Superstar wie Prince, sondern auch für (noch) unbekannte Künstler möglich. Mit Sampler und Sequenzer lassen sich die Beats der Stücke in Eigenregie produzieren; für bestimmte Instrumentalfarben werden Studio-Musiker engagiert - nicht selten Jazzmusiker wie zum Beispiel Roy Hargrove bei D'Angelos Voodoo oder Ron Carter bei Erikah Badus Debüt Baduism (1997) - oder aber die eigenen instrumentalen Fähigkeiten eingesetzt (wie bei den Alben von D'Angelo). Tobias Lindemann und Tine Plesch (2004: 111f.) haben darauf hingewiesen, dass die durch Sampler und Sequencer-Software allgemein verfügbare Homestudio-Technologie zur künstlerischen Emanzipation gerade der R\&B-Frauen beigetragen habe, die zuvor auf die fast ausschließlich männlichen Musiker und Produzenten angewiesen waren. Ein frühes Beispiel für eigenverantwortlich produzierende R\&B-Frauen ist Anita Baker mit ihrem Album Rapture (1986), das der afroamerikanische Musikjournalist Nelson George (1986: 186ff.) zugleich als ein Beispiel des so genannten "Retronuevo « bezeichnete - einer Stilrichtung afroamerikanischer Musik, die in der Tradition des Soul und des »Quiet Storm«-Late Night-Radioformats ${ }^{4}$ steht, sich durch ein vorwiegend akustisches Instrumentarium vom synthetischen Sound der 1980er Jahre abhebt und dabei viele Elemente des Neo-Soul der 1990er und 2000er Jahre vorwegnimmt.

Doch zurück zu Lauryn Hill und ihrem Erfolgsalbum The Miseducation of Lauryn Hill. Wie in vielen Rap Music-Aufnahmen seit den 1990er Jahren üblich, verbindet Hill in der Hit-Auskopplung »Doo Wop (That Thang)« RapStrophen mit einem gesungenen Refrain. Auffällig ist der Break vor dem Schlussrefrain, in dem aus mehreren gleichzeitig erklingenden Aufnahme-

4 Die Bezeichnung "Quiet Storm « für eine klanglich vergleichsweise sanfte Spielart des Soul, die seit Mitte der 1970er Jahre im Nachtprogramm afroamerikanischer Radio-Stationen gesendet wird, entstammt dem Titel der 1975 erschienenen LP A Quiet Storm von Smokey Robinson. 
spuren eine dichte Textur von Gesangsstimmen gewoben wird - was insbesondere durch die Bass-Stimme (»boom boom«) eine Reminiszenz an den mehrstimmigen Gesang der DooWop-Bands der 1950er Jahre darstellt.

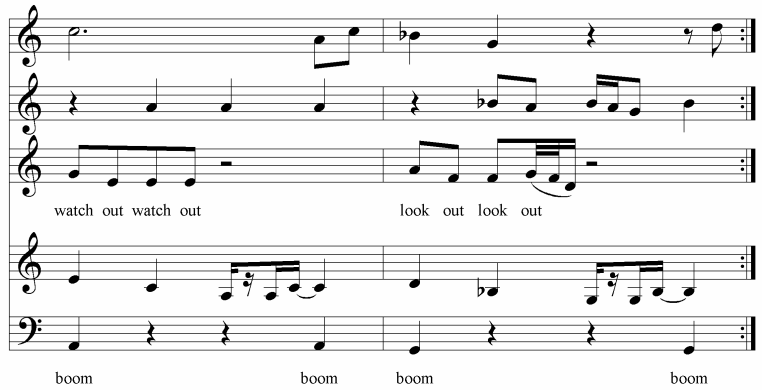

Abbildung 1: Lauryn Hill - »DooWop (That Thing)«, Vocal-Break vor Schlussrefrain

In der überwiegenden Zahl der Stücke ihres Albums verzichtet Hill allerdings völlig auf Raps und stellt ihren Gesang in den Mittelpunkt. Zentrales Kennzeichen des Gesangsstils von Lauryn Hill - und generell von vielen Vokalisten des neueren R\&B - ist eine ausgeprägte Melismatik, also das ornamentierende Aussingen einzelner Textsilben. Ein typisches Beispiel hierfür ist Hills »When It Hurts So Bad«. Eingerahmt wird der Song von einer Introduktion und einer Coda mit dem Text »When it hurts so bad, why does it feel so good?«. Der Text in den beiden Strophen berichtet von einer unglücklichen Liebeserfahrung, während im Refrain eine Art Lehre aus dieser Erfahrung gezogen wird:

What you want might make you cry

What you need might pass you by

If you don't catch it

And what you need ironically

Will turn out what you want to be

If you just let it

(aus Lauryn Hill: »When It Hurts So Bad «)

Das Tempo des Songs ist langsam (80 bpm); die Harmonik ist mit zwei viertaktigen Vamps über Dm / Em bzw. Em / Am einfach gehalten. Die Instrumentierung ist mit Harfe, akustischer Gitarre, Bass und einem reduzierten Schlagzeugset (nur Bass-Drum und Snare) sowie Claves und gelegentlich Bongos klanglich eher unaufdringlich und zurückhaltend; die SchlagzeugBreaks sind den Breaks im jamaikanischen Reggae nachempfunden. ${ }^{5}$ Auf-

5 Anklänge an Reggae finden sich bereits in Aufnahmen des Rap-Trios The Fugees. 
fällig sind auch in diesem Stück die zahlreichen Gesangsspuren: In vielen Passagen wird eine Hauptstimme durch mehrere leisere Gesangsstimmen ergänzt. Die Transkription der Gesangsstimme in der ersten Hälfte der zweiten Strophe versucht, die stark melismatische Gestaltung als Notentext darzustellen.
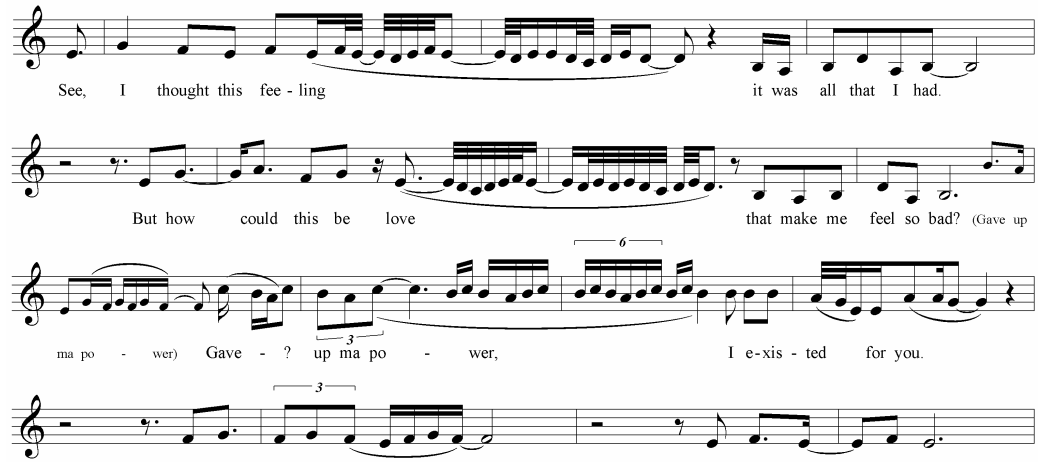

But who - e - ver knew

the voo - doo you'd do

Abbildung 2: Lauryn Hill - »When It Hurts So Bad«, 2. Strophe, Zeile 1-4

In der ersten Zeile verziert Hill ausgiebig die zweite Silbe des Wortes »feeling «, indem sie zwischen den Tönen $c, d, e$ und $f$ hin- und hergleitet. In den folgenden Zeilen sind es die Wörter »love«, »power« und »knew«, die von Hill melismatisch in einem relativ engen Ambitus ausgestaltet werden. Durch die Ornamentik werden somit die besonders gefühlsbeladenen Schlüsselwörter des Textes in den Vordergrund gerückt. Zu Beginn der dritten Zeile (»Gave up ma power«) nimmt zudem eine leisere Stimme (in Abbildung 2 kleiner gedruckt) den Einsatz der Hauptstimme vorweg, deren Text in den ersten Silben unverständlich ist.

Die Melismen sind vom metrischen Bezugspunkt weitgehend losgelöst die Transkription ist dabei nur als ein Annäherungsversuch an die genaue zeitliche Gestaltung zu verstehen. Dagegen wird die Schlussphrase der einzelnen Zeilen jeweils synchron mit der Schlagfolge der Begleitinstrumente gesungen. Hill löst sich innerhalb der Gesangsphrase vom metrischen Zeitraster der Begleitmusiker, lässt sich also bei der melodischen Gestaltung entspannt Zeit, kommt quasi »zu spät« und doch zu den entscheidenden Bezugspunkten am Phrasenende präzise »auf den Punkt«.

Die Melismatik der Gesangsstimme besteht nicht, wie der Notentext womöglich suggeriert, aus einer Abfolge von gegeneinander abgegrenzten Klängen fester Tonhöhe, sondern vielmehr aus einem stufenlosen Gleiten 
zwischen den Tonhöhen, die nur Umkehrpunkte bzw. Durchgangsstadien einer kontinuierlichen Melodiebewegung sind, sowie aus einem Hineingleiten in die Tonhöhe, besonders auffällig bei »power in der dritten Zeile. Diese zwischen den Tonhöhen gleitende Singweise wird in der Spektraldarstellung der Musikausschnitte besonders augenscheinlich (Abbildung 3 ).

In den Spektrogrammen ist der Verlauf der Gesangsstimme als parallele waagrechte Linien zu erkennen, wobei die unterste Linie die Frequenz des Grundtons des gerade gesungenen Vokals, die höher gelegenen Linien die Frequenzen der verschiedenen Partialtöne darstellen. Die regelmäßigen Impulse des Schlagzeugs sind aufgrund ihres breiten Frequenzspektrums und ihrer kurzen Dauer als vertikale Linien zu erkennen; dabei sind die etwas dickeren und dunkleren Balken, die in regelmäßigen Abständen auftauchen, den stärkeren Snare-Schlägen (in der Regel auf 2 und 4) zugeordnet. ${ }^{6}$ Der dargestellte Frequenzbereich wurde mit 20 bis $5500 \mathrm{~Hz}$ relativ klein gewählt, damit die Tonhöhenbewegung klarer zu erkennen ist; er deckt jedoch das Klangspektrum der Stimme weitgehend ab. Nur in der dritten Zeile wurde ein weiter Frequenzbereich $(20 \mathrm{~Hz}$ bis $10 \mathrm{kHz}$ ) gewählt, wodurch die höheren Frequenzanteile des laut und »strahlend « gesungenen Wortes »power « sichtbar werden. Zusätzliche waagrechte Linien zeugen entweder von Begleitinstrumenten, oder aber von weiteren Gesangsstimmen.

Lauryn Hills Gesangsstil knüpft an die Tradition des Gospelgesangs an. In der afroamerikanischen Gospel Music findet sich eine ausgeprägte Silbendehnung und Melismatik, die etwa von der einflussreichen Soul-Sängerin Aretha Franklin sowohl in ihren Gospel-Aufnahmen, so in dem Konzertmitschnitt »Amazing Grace« (1972) (vgl. Putschögl 1998), als auch in etwas abgeschwächter Form in vielen Soul-Aufnahmen eingesetzt wurde. Vergleichbar ausgeprägte Melismen wie bei Hill finden sich in den Aufnahmen zahlreicher anderer Neo-Soul-Künstler, so bei D'Angelo (stark ausgeprägt z.B. in »Sent It On« auf Voodoo, 2000) oder Bilal Oliver (z.B. in »Fast Lane « auf 1st Born Second, 2001). Der Effekt einer endlos gleitenden Stimme wird bei vielen Aufnahmen von Hill, D'Angelo, Bilal Oliver und anderen R\&B- Sängerinnen und -Sängern dadurch noch verstärkt, dass mehrere melismatisch

6 Die Spektraldarstellungen wurden mit einer Demo-Version der KlanganalyseSoftware STx 3.6.1 erstellt, die vom akustischen Forschungsinstitut der österreichischen Akademie der Wissenschaften entwickelt worden ist. Dabei wurde auf das Analyseverfahren der schnellen Fouriertransformation (FFT) mit einem Analysefenster von $33 \mathrm{~ms}$ (oder 1470 Samples bei $44.1 \mathrm{kHz}$ ) und einer 70-prozentigen Überlappung der Analysefenster zurückgegriffen. Hieraus resultiert eine Frequenzauflösung in Bändern von $30 \mathrm{~Hz}$, die in einem Frequenzbereich von 20 bis $5500 \mathrm{~Hz}$ und einem Lautstärkebereich von -15 bis $-75 \mathrm{~dB}$ als unterschiedlich starke Graustufen dargestellt werden. 

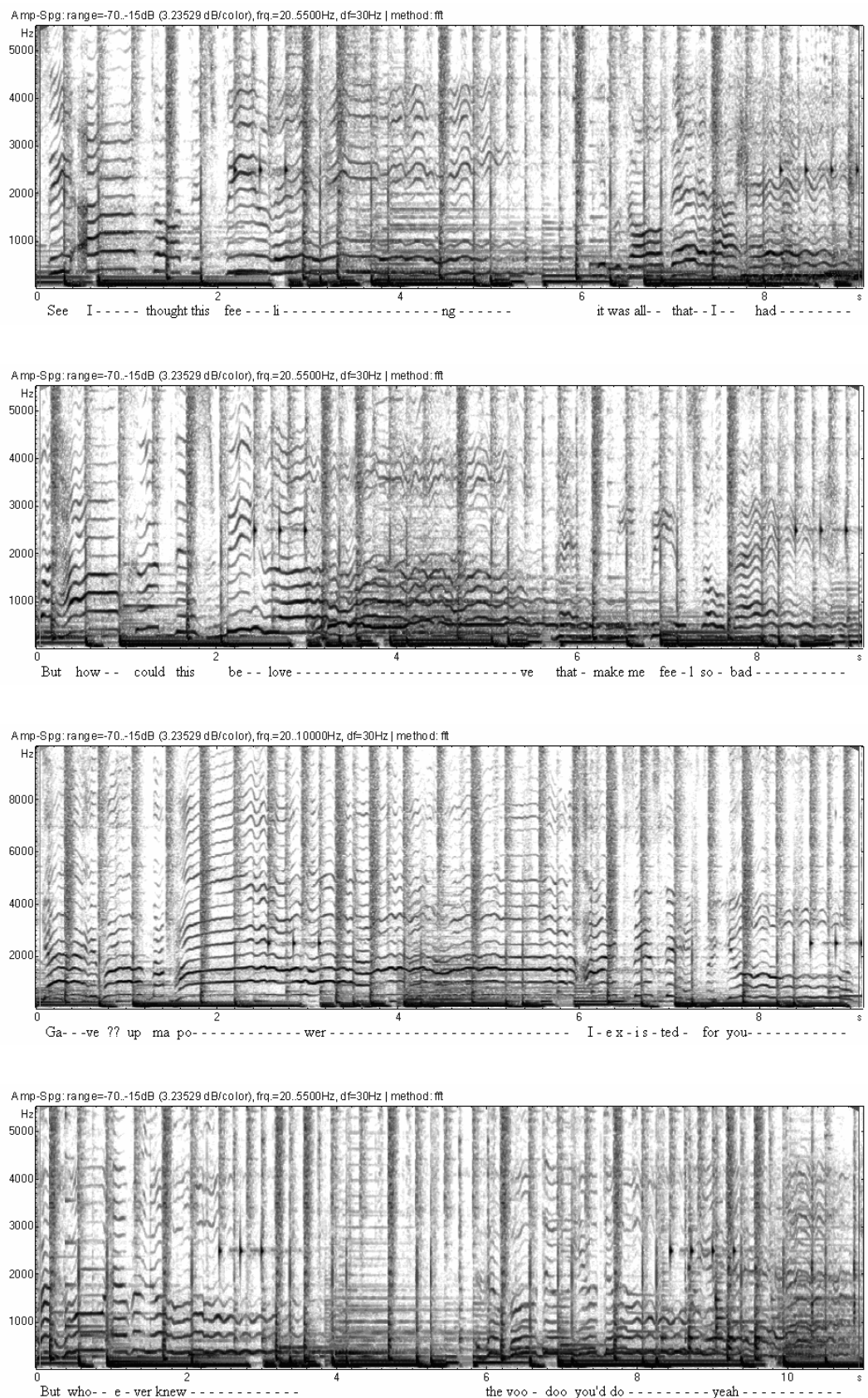

Abbildung 3: Lauryn Hill - »When It Hurts So Bad«,

Spektraldarstellungen der 2. Strophe, Zeile 1-4 
gestaltete Gesangsspuren gleichzeitig erklingen. Diese Gesangsstimmen werden vom Vokalisten per Multiplayback-Verfahren nacheinander aufgenommen. Bei Konzertauftritten werden sie mitunter durch mehrere Background-Sängerinnen und -Sänger reproduziert. Auch in »When It Hurts So Bad « kommt dieses Aufnahmeverfahren zum Einsatz; so etwa in der zweiten Zeile bei der Silbe »love«, was auch im Spektrogramm gut zu erkennen ist. Ähnlich wie in »DooWop (That Thang) « wird am Schluss des Songs ein Durchgang des Refrains von mehreren Gesangsstimmen vorgetragen und nur von einer akustischen Gitarre begleitet; dabei erklingt die Refrainmelodie mit der lautesten Gesangsspur, während die anderen Stimmen die Melodie improvisatorisch verzieren und umranken. Die Verdopplung, Wiederholung und variierende Ausgestaltung von Wörtern und Phrasen mit Hilfe der modernen Mehrspurtechnik knüpft an die ebenfalls im Gospelgesang verbreitete textliche Ausschmückung durch die Sängerin oder den Sänger mit Hilfe von verstärkenden Adjektiven, Substantiven und eingeschobenen Sätzen sowie "Silben-, Wort- oder Phrasenwiederholungen, Silbendehnungen und Melismatik« (Putschögl 1998: 343) an. Ein an diesen Gestaltungsmitteln orientierter Gesangsstil ist spätestens seit Aretha Franklin bei vielen Vokalisten nicht nur im R\&B üblich geworden, da er eine emotionale Intensität, Ernsthaftigkeit und Innigkeit zum Ausdruck bringt, wie sie ja in der populären Musik angesichts der vorherrschenden Authentizitäts-Ideologie (Frith 1987) allenthalben gefragt ist. Diese Gestaltungsmittel bilden inzwischen auch einen festen Bestandteil des Gesangsstils von Mainstream-Pop-Größen wie Britney Spears, Christina Aguilera u.a.

Liest man in biographischen Notizen über Sängerinnen und Sänger des neueren R\&B, so bildet die Erfahrung der Black Church, der afroamerikanischen Gottesdienste, einen festen Bezugspunkt in der Sozialisation vieler Künstler. Einige von ihnen sangen selbst als Kinder und Jugendliche in der Kirche. Ein weiterer Bezugspunkt ist die Tradition des Soul und Funk, die sich die zumeist erst in den 1970er Jahren geborenen Künstler über Plattensammlungen von Eltern oder Verwandten erschlossen und angeeignet haben. Die ersten eigenen musikalischen Aktivitäten erfolgten dagegen vielfach, wie bei Lauryn Hill oder D'Angelo, im Bereich der Rap Music, während der R\&B- und Soul-Gesang erst später ins Zentrum des eigenen musikalischen Schaffens rückte. Neben Aretha Franklin sind dabei vor allem jene Sänger einflussreich, die sich wie Marvin Gaye oder Curtis Mayfield durch eine hohe Stimmlage bzw. den gezielten Einsatz der Kopfstimme auszeichnen. Dieser Gesangsstil wird im Gegensatz zur vollen, »schmutzigen« Gesangsweise von Soul-Sängern wie z.B. Wilson Pickett oder den kurzen, abgehackten Schreien 
von James Brown nicht mit Machismo und männlicher Aggressivität, sondern mit Weichheit und Verletzlichkeit assoziiert.

Fragen der Geschlechterrollen und der Männlichkeit werden auch in dem zweiseitigen Text thematisiert, den D'Angelo im Cover seiner zweiten, in Eigenregie produzierten CD Voodoo (2000) veröffentlicht hat. "If we are to exist as men in this new world «, so schreibt der damals 25-jährige Sänger (D'Angelo 2000), »many of us must learn to embrace and nurture that which is feminine with all of our hearts (he-art). « D'Angelos Text beinhaltet weitere Motive, die im Kontext der gegenwärtigen afroamerikanischen Musik immer wieder thematisiert werden. Er beschwört die herausragenden Künstler der populären afroamerikanischen Musiktradition - Jimi Hendrix, Sly Stone, Al Green, Marvin Gaye, Stevie Wonder, Prince - und stellt seine Kunst in einen quasi-religiösen Zusammenhang: "Art is the word that connects the individual (thou) to their higher self (God) or to that which is universal « (ebd.). Breiten Raum nimmt außerdem die Frage nach der Abgrenzung zwischen Kunst und Kommerz bzw. Business ein. Letzteres macht D'Angelo sowohl an den HipHop-Stars mit ihren Goldketten als auch am Schaffen von Prince fest und führt dagegen die Kriterien Handwerk (»craft «) und Inspiration ins Feld.

Man kann D'Angelos Text als programmatischen Versuch der Identitätskonstruktion begreifen, als Selbstvergewisserung innerhalb eines Felds von unterschiedlichen Typen afroamerikanischer Identität. Nelson George hat bereits Anfang der 1990er Jahre darauf hingewiesen, dass seit den 1970er und 1980er Jahren in der Post-Soul-Kultur eine Reihe neuer afroamerikanischer Identitätstypen entstanden sind:

»There is the Buppie, ambitious and acquisitive, determined to savor the fruits of integration by any means necessary; the B-boy, molded by hip hop aesthetics and the tragedies of underclass live; the Black American Princess or Prince a/k/a Bap, who, whether by family heritage or personal will, enjoys all expectation of mainstream success and acceptance that borders on arrogance; and the Boho, a thoughtful, self-conscious figure [...], whose range of interest and taste challenges both black and white stereotypes of African American behaviour « (George 1992: 2).

Gerade der B-Boy-Stil, so George weiter, wirkt weit über seine GhettoUrsprünge hinaus und beeinflusst nachhaltig Sprache, Kleidung und Musik der Schwarzen, so dass sich die anderen drei Typen bewusst in Abgrenzung zu dieser Herausforderung konstruieren. Die Reaktion auf die HipHop-Kultur fällt bei D'Angelo - und ebenso bei vielen anderen zeitgenössischen R\&BKünstlern - ambivalent aus: Einerseits äußert er Kritik an der Oberflächlichkeit, Kommerzialität und der mangelnden Inspiration vieler Rapper; 
andererseits nähert er sich in der Kleidung und durch die Einbeziehung von HipHop-Beats, Raps und Rappern in die eigene Musik - auf Voodoo ist es der Rapper Method Man vom Wu Tang Clan - der vorherrschenden B-BoyAttitüde an. Ähnlich zwiespältig ist die Auseinandersetzung mit dem so genannten Bap, der einerseits in Person des Crossover-Stars Prince kritisiert wird (»he lacks any new inspiration«, D'Angelo 2000); andererseits sieht sich D'Angelo gehalten, selbst auf den Vorwurf zu reagieren, er wolle nach dem überragenden kommerziellen Erfolg seiner Debüt-CD Brown Sugar ${ }^{7}$ (1995) mit Voodoo nur einfach seinen Profit verdoppeln. Die Beschwörung der Tradition afroamerikanischer Musik und einer Art Kunstreligion kann als Strategie verstanden werden, die eigene Ernsthaftigkeit und Authentizität unter Beweis zu stellen. Unterstrichen wird dies musikalisch durch den Rückgriff auf jene vokalen Gestaltungsmittel aus der afroamerikanischen Gospel- und Soul-Tradition, die dort Ernsthaftigkeit, religiöse Ergriffenheit und emotionale Intensität ausdrücken.

Mark Anthony Neal hat in Anknüpfung an die Typologie von Nelson George auf die Durchlässigkeit zwischen den neuen afroamerikanischen Identitätstypen hingewiesen:

»While we embrace these identities as part of our being, we are also conscious of the fluidity of the communities to which we belong and the relative freedom to explore these identities often playfully at the expense of white onlookers, in ways that our parents could never conceive« (Neal 2002: 177).

Vor diesem Hintergrund wird sich die Erforschung gegenwärtiger Tendenzen in populärer afroamerikanischer Musik verstärkt mit den Mitteln und Strategien auseinandersetzen müssen, mit denen einerseits Authentizität konstruiert und andererseits unterschiedliche Identitäten spielerisch miteinander vermischt werden.

7 Tatsächlich bezieht sich D'Angelos Song »Brown Sugar «, der den Titel für meinen Aufsatz lieferte, weder auf die Farbe der in R\&B-Videoclips reichlich zur Schau getragenen Haut noch auf den »süßlichen« R\&B-Gesang, sondern auf D'Angelos bevorzugte Haschisch-Sorte (»that's why my eyes are a shade blood burgundy«); vgl. Shapiro 2000: 38. 


\section{Literatur}

Bogdanov, Vladimir / Erlewine, Stephen Thomas / Bush, John / Woodstra, Chris (Hg.) (2003). All Music Guide to Soul: The Definitive Guide to R\&B and Soul. San Francisco: Backbeat Books.

Brackett, David (1994). "The Politics and Practice of >Crossover < in American Popular Music, 1963 to 1965. «In: Musical Quarterly 78, S. 774-797.

Bynoe, Yvonne (2002). »Money, Power, and Respect: A Critique of the Business of Rap Music. In: R\&B. Rhythm and Business: The Political Economy of Black Music. Hg. v. Norman Kelly. New York: Akashic Books, S. 220-234.

Frith, Simon (1987). »Towards an Aesthetic of Popular Music. In: Music and Society. The Politics of Composition, Performance and Reception. Hg. v. Richard Leppert und Susan McClary. Cambridge: Cambridge University Press, S. 133-149.

George, Nelson (1988). The Death of Rhythm and Blues. New York: E. P. Dutton.

George, Nelson (1992). Buppies, B-Boys, Babs \& Bohos: Notes on Post-Soul Black Culture. New York: Harper.

Henderson, Alex (2003). »Neo-Soul. «In: Bogdanov et al. 2003: S. 855f.

Larkin, Colin (1998). The Virgin Encyclopedia of R\&B and Soul. London: Virgin.

Lindemann, Tobias / Plesch, Tine (2004). "Queens and Divas. Rhythm'n'Blues zwischen Kollektivtraditionen, Individualitätsmythen und Geschlechterpolitik. « In: Testcard 13. Black Music, S. 105-113.

Neal, Mark Anthony (2002). Soul Babies: Black Popular Culture and the Post-Soul Aesthetic. New York: Routledge.

Putschögl, Gerhard (1998). »Zu den rhythmisch freien Gestaltungsformen der afroamerikanischen Musik am Beispiel John Coltranes. " In: ...und der Jazz ist nicht von Dauer. Aspekte afro-amerikanischer Musik. Festschrift für Alfons Michael Dauer. Hg. v. Bernd Hoffmann und Helmut Rösing (= Forum Jazz Rock Pop 1). Karben: Coda, S. 333-369.

Schloss, Joseph G. (2004). Making Beats: The Art of Sample-Based Hip-Hop. Middletown, CT: Wesleyan University Press.

Shapiro, Peter (2000). Soul. 100 Essential CD's: The Rough Guide. London: Rough Guide.

Ward, Brian (1998). Just My Soul Responding: Rhythm and Blues, Black Consciousness, and Race Relations. Berkeley: University of California Press.

\section{Diskographie}

D'Angelo (2000). Voodoo. Cheba Sound/EMI, 724352337323.

Hill, Lauryn (1998). The Miseducation Of Lauryn Hill. Ruffhouse/Columbia, 4898432. 


\begin{abstract}
This paper discusses aspects of Rhythm'n'Blues in the late 1990s and early 2000s, when neo-soul artists like Lauryn Hill, D'Angelo or Mary J. Blige gained wider success. Since the 1990s the borders between rap music and contemporary R\&B have become increasingly unclear, as in both genres the same production strategies with samplers and sequencers are often used by the same producers, creating similarly sounding beats (though R\&B-artists sometimes prefer acoustic instruments and jazzy sounds). Personal collaborations between rap artists and R\&B-singers are not unusual. The main characteristic of new R\&B is a richly ornamented or melismatic singing style as shown by the example of Lauryn Hill. Finally, thoughts on artistry and identity by R\&B-singer D'Angelo are connected with thoughts on African American post-soul identities.
\end{abstract}

